\title{
A CRC-Aided Hybrid Decoding Algorithm for Turbo Codes
}

\author{
Yuejun Wei, Ming Jiang, Bin Xia, Wen Chen, and Yuhang Yang
}

\begin{abstract}
In this letter, we propose a hybrid turbo decoding algorithm, where the outer code, CRC code, is not used for error detection as usual but for error correction improvement. The algorithm combines the iterative decoding with ordered statistics decoding (OSD), where the CRC code and the turbo code are regarded as an integrated whole in the OSD process. Moreover, we propose an effective error detecting method based on normalized Euclidean distance to compensate for the loss of error detection capability which should have been provided by CRC. Simulation results show that with the proposed approach, $0.5-2 \mathrm{~dB}$ performance gain can be achieved for the code blocks with short information length.
\end{abstract}

Index Terms-Turbo codes, cyclic redundancy check, ordered statistics decoding, normalized Euclidean distance.

\section{INTRODUCTION}

$\mathbf{T}$ URBO codes [1] have been adopted in the long-term evolution (LTE) systems due to the fact that they can not only achieve high throughput with their parallel decoding architecture, but also support almost any code rate and arbitrary code block (CB) length from 40 bits to 6144 bits for various services in LTE. However, turbo codes usually suffer severe performance degradation for services with short $\mathrm{CB}$ length, e.g., the voice over Internet protocol (VoIP) service [2], where the CB length is limited from 40 bits to about 352 bits.

In LTE, there are always 24 cyclic redundancy check (CRC) bits attached after the information bits in the physical layer, where the mixed bit stream to be encoded forms a CB. The CRC codes add considerable overhead which dramatically decreases the transmission efficiency, when the CB length is short. For example, the penalty of the coding gain caused by the $24 \mathrm{CRC}$ bits almost achieves $4 \mathrm{~dB}$ when the information length before CRC encoder is 16 and the CB length is 40. As we can see, due to the obvious CRC overhead for short CB lengths, there exists an inherent performance gap between the realistic iterative turbo decoding and the maximum likelihood decoding (MLD) for concatenated turbo-CRC codes.

Besides error detection, some literature shows that CRC codes can also assist error correction during the channel decoding process. Correction impulses and repeated (CIR) decoding [3] and soft list Viterbi algorithm (SLVA) assisted channel decoding [4] efficiently reduce the performance gap

Manuscript received February 3, 2013. The associate editor coordinating the review of this letter and approving it for publication was G. Colavolpe.

Y. Wei, B. Xia, W. Chen, and Y. Yang are with the Department of Electronic Engineering, Shanghai Jiao Tong University, Shanghai, China (e-mail: \{yjwei, bxia, wenchen, yhyang\}@sjtu.edu.cn).

M. Jiang is with the National Mobile Communications Research Laboratory, Southeast University, Nanjing, China (e-mail: jiang_ming@seu.edu.cn).

This work is supported by the National 973 Project \#2012CB316106 and NSF China \#61161130529.

Digital Object Identifier 10.1109/WCL.2013.052813.130259 between the conventional decoding and the MLD. However, their contributions mainly lie in the improvement on the error floor. In [5], CRC codes are involved in the iterative decoding, where they are considered as the component codes serially concatenated with the convolutional codes. Unfortunately, the parity check matrices (PCM) of the classic CRC codes are not appropriate for the iterative decoding, since the density of PCM is not sufficiently sparse and 4-cycle-free assumption can not be guaranteed.

In this letter, we propose a CRC-aided hybrid decoding for turbo codes, where the decoding scheme combines the iterative-based standard turbo decoding (STD) with the ordered statistics decoding (OSD) [6], [7]. The hybrid decoding of the concatenated turbo-CRC codes incorporates the CRC bits into the OSD process to further lower the error probability. Since the CRC bits participate in the error correction process and lose the error detection ability, we propose an error detection approach based on the normalized Euclidean distance (NED). Simulation results show that the proposed CRC-aided hybrid decoding scheme can significantly improve the performance of turbo codes with short CB length.

\section{The Hybrid Decoding of the Turbo Codes}

The two component codes in the LTE turbo codes are both recursive systematic convolutional (RSC) codes with the same generator polynomial $[1,13 / 15]$. We can transform the polynomial of the RSC codes into an infinite periodic polynomial, where the coefficients can be defined as an infinite binary sequence, $\mathbf{A}=\{1, \mathbf{a}, \mathbf{a}, \mathbf{a}, \ldots .$.$\} , with \mathbf{a}=[1110010]$. Then, we can get a vector $\mathbf{p}_{0}$ by selecting the first $k$ elements from $\mathbf{A}$ in line with the $\mathrm{CB}$ length. A $k$-by- $k$ matrix $\mathbf{P}_{k}$ can be constructed using a right shift operation on the vector $\mathbf{p}_{0}$ row by row. Finally, we obtain the generator matrix $\mathbf{G}^{\text {turbo }}=\left[\mathbf{I}_{k}\left|\mathbf{P}_{k}\right| \tilde{\mathbf{P}}_{k}\right]$, where the identity matrix $\mathbf{I}_{k}$ and the matrix $\mathbf{P}_{k}$ (the interleaved one as $\tilde{\mathbf{P}}_{k}$ ) are corresponding to the systematic bits and the parity bits, respectively. The sub-matrix $\tilde{\mathbf{P}}_{k}$ can be obtained by permuting the rows of the matrix $\mathbf{P}_{k}$ according to the quadratic permutation polynomial (QPP) interleaver [1].

The polynomial of the LTE CRC (named CRC24a in [1]) is $\mathrm{g}^{\mathrm{CRC}}(D)=\sum_{i=0}^{24} g_{i} \times D^{i},\left\{g_{24}, g_{23}, \ldots, g_{0}\right\}=$ $\{1100001100100110011111011\}$. The information length is $m=k-24$ and the entire generator matrix of the turbo-CRC code is the product of the two generator matrices of the CRC encoder and the turbo encoder,

$$
\mathbf{G}=\mathbf{G}^{\mathrm{CRC}} \times \mathbf{G}^{\text {turbo }} .
$$

At the receiver, the reliability-based OSD and the iterativebased STD can be carried out individually and collaboratively. These two decoding processes are proposed based on the 
different principles in error correction. Hence, both the STD and the OSD can be carried out independently on the received signals $Y=\left\{y_{1}, \cdots, y_{3 k}\right\}$. For the zero-tailing technique used in the LTE turbo codes, because the LLRs of tail bits are not updated during the iterative decoding, they usually are not as reliable as the LLRs of the systematic bits and the parity bits updated after a number of iterations. Therefore, we do not consider the tail bits in the OSD process. However, some special schemes for the termination of turbo codes would also support the inclusion of the tail bits in the processing.

The OSD process comprises three stages: sorting, Gaussian elimination and re-encoding. Firstly, the hard-decision bits based on the signs of the input information $\left\{R_{1}, \cdots, R_{3 k}\right\}$ and the columns of the $\mathbf{G}^{\text {turbo }}$ are both permuted according to the reliability $\left\{\left|R_{1}\right|, \cdots,\left|R_{3 k}\right|\right\}$ sorted in decreasing order. Then, the Gaussian elimination is applied to the permuted matrix to obtain the systematic generator matrix $\tilde{\mathbf{G}}^{\text {turbo }}$. Since the $\mathrm{k}$ most reliable columns of the generating matrix could be not linearly independent, they must be changed by involving the other $2 k$ columns in this case. Finally, the re-encoding is executed according to the $\tilde{\mathbf{G}}^{\text {turbo }}$ and the permuted harddecision bits, where the CRC detection is performed based on the first reverse-permuted $k$ bits in the re-encoded codeword [6]. In order to improve the performance, the high order OSD process can be carried out, where the order- $N$ OSD flips of all subsets of the hard-decision bits with length less than $N$ and performs re-encoding to provide a collection of candidate codewords.

In the hybrid decoding, after each iteration of STD, not only the log-likelihood-ratios (LLRs) of the systematic bits, but also those of the parity bits should be calculated for the hybrid decoding. The updated LLRs of both the systematic bits and the parity bits after the $t$-th iteration are denoted as $\left\{L_{1}^{t}, \cdots, L_{3 k}^{t}\right\}$. The updated LLRs after each iteration of the STD can provide more reliable information, so the OSD can be efficiently performed using the output LLRs of the $t$-th iteration in STD, $\left\{R_{i}=L_{i}^{t}, i=1, \cdots, 3 k\right\}$. A parameter $f$ is selected to indicate the iteration after which the OSD process is started. For example, if the parameter $f$ is set to 1 , the OSD process is performed after each iteration; if $f$ is set to $T$, i.e., the maximum number of iterations, the OSD process is performed only after the last iteration of the turbo decoding.

As LLR oscillation is an inevitable problem during the iterative turbo decoding [8], the magnitudes of the updated LLRs in the current iteration are not always reliable for the OSD process. In order to overcome the LLR oscillation, an accumulation of LLRs is proposed in [9] for the OSD process of low density parity check codes. After $t, 1 \leq t \leq T$ iterations, the accumulated LLRs are set to be the inputs of the OSD, which can be calculated as

$$
R_{i}^{t}=L_{i}^{t}+\alpha R_{i}^{t-1}, i=1, \ldots, 3 k,
$$

where the initialized accumulated LLRs, $\left\{R_{1}^{0}, \cdots, R_{3 k}^{0}\right\}$, are set to zero. The accumulated LLRs are equal to the updated LLRs in each iteration when the accumulation parameter $\alpha$ is set to zero.

The typical value of the accumulation factor $\alpha$ varies according to the value of $f$. When the OSD process is carried out after each iteration (i.e., $f=1$ ), we intend to test the multiple OSD processes with the input information as different as possible to get more "diversity" gain. Then, the absolute values of the current output LLRs should be directly used without accumulation operation, i.e., $\alpha=0$. On the other hand, if the OSD is only performed after the last iteration of the STD (i.e., $f=T$ ), the output LLRs of all the iterations are fully accumulated, i.e., $\alpha=1$, in order to obtain more reliable information. When the OSD is not applied at the end of the turbo decoding but in the middle, i.e., $f<T$, the STD process will not stopped until the maximum number of iteration is reached. If the STD result still cannot pass the CRC after $T$ iterations, the codeword of OSD with the minimum Euclidean distance among all OSD results is selected as the hybrid decoding result.

\section{ThE CRC Assistance In ThE OSD PROCESS}

In the CRC-aided hybrid decoding scheme, the CRC codes are treated as component codes of the concatenated turboCRC codes and make extra contributions to error correction. We replace the matrix $\mathbf{G}^{\text {turbo }}$ with the entire generator matrix of the turbo-CRC codes $\mathbf{G}$ in (1) for the OSD process, incorporating the additional constraints introduced by the CRC codes in decoding. With the assistance of the CRC codes, the hybrid decoding can further narrow the performance gap to the MLD of turbo-CRC codes with $k-24$ information bits, when the Gaussian elimination and re-encoding in the OSD process are carried out.

Since the matrix $\mathbf{G}$ in (1) already incorporates the CRC generator matrix, all the re-encoded codewords from the OSD process based on the matrix $\mathbf{G}$ always satisfy the CRC. We can only select the codeword with the minimum Euclidean distance to the received signals as the decoding result. Assuming that order- $N$ re-encoding is performed and there are total $\Phi(N)=\left(\begin{array}{l}k \\ N\end{array}\right)$ codewords generated, the computation of Euclidean distance for the $n$-th codeword $C^{n}=\left\{c_{1}^{n}, \cdots, c_{3 k}^{n}\right\}$ can be calculated by the approximate method proposed in [10]

$$
d^{n}=\sum_{c_{i}^{n} \neq z_{i}, i=1, \cdots, 3 k}\left|y_{i}\right|, n=1, \cdots, \Phi(N),
$$

where the sequence $\left\{z_{1}, z_{2}, \cdots, z_{3 k}\right\}$ is the hard decision of the received signals. The codeword $C^{*}$ which has the minimum distance

$$
d^{*}=\min _{n=1, \cdots, \Phi(N)} d^{n}
$$

is regarded as the final result, when the STD fails after $T$ iterations.

In the CRC-aided hybrid decoding, since the CRC bits have participated in the decoding process and lost the error detection ability, there is always a "valid" output from the OSD process for the turbo-CRC codes. Therefore, the increase of the undetected error rate (UER) is unavoidable, which will lead to serious degradation of the system throughput. To compensate for the deficiency in error detection, we propose an alternative error detection criterion based on the NED. The NED metric of the codeword $C^{*}$ is calculated as follows,

$$
\hat{d}^{*}=\frac{d^{*}}{\sum_{i=1, \cdots, 3 k}\left|y_{i}\right|} \text {. }
$$


A threshold $\eta$ can be set for error detection. If the NED metric of the output codeword $C^{*}$ from the OSD is larger than the preset threshold, i.e., $\hat{d}^{*}>\eta$, the hybrid decoding result is regarded as an error, otherwise correct decoding is assumed. Not only the undetected error but also the false alarm probability should be considered when optimizing the threshold $\eta$. Although the error detection ability of the NED criterion is not as good as that of the 24-bit CRC, the UER of the NED criterion with the optimized $\eta$ can still achieve near one thousandth of the block error rate (BLER), which will be shown in the simulation results. Furthermore, the normalization makes the NED independent of the scaling of the received signals. Simulation results also show that the suitable value of the threshold $\eta$ is independent of the signalto-noise ratio.

\section{Complexity Analysis of the Hybrid Decoding}

Because the key process of the OSD, the Gaussian elimination of the generator matrix, has a cubic complexity, there are approximately $O\left(k^{3}\right)$ binary addition and $O\left(k^{2}\right)$ real additions in the order-1 OSD process [6]. The complexity of the OSD usually is much higher than that of the STD whose complexity is at the level of $\mathrm{O}(\mathrm{k})$. Fortunately, the proposed hybrid decoding is mainly applied for the turbo codes with short information length. When the information length is less than or of order 328, i.e., the typical lengths for the VoIP services, one order-1 OSD process can be executed in $1.6 \times 10^{5}$ cycles in our FPGA platform with $200 \mathrm{MHz}$ frequency, where the total decoding latency is less than $1 \mathrm{~ms}$ which is acceptable for a lot of applications such as VoIP [2].

Moreover, the CRC-aided scheme can reduce the dimension of the generator matrix, which leads to a lower complexity of the Gaussian elimination. For example, there is $k-24$ rows in the generator matrix $\mathbf{G}$ of the concatenated turbo-CRC code, which is less than that of the original $k$ rows in the matrix $\mathrm{G}^{\text {turbo }}$ due to the multiplication of the matrix $\mathbf{G}^{\mathrm{CRC}}$ with 24 rows. Therefore, the CRC assistance in the OSD process results in noticeable decrease in the complexity of Gaussian elimination $O\left((k-24)^{3}\right)$, when the information length $k$ of the turbo code is short.

After the $t$-th turbo iteration, the higher order OSD process can also be employed to further improve the error performance, where the re-encoding process is performed with higher reliable combinations of the $k$ hard-decision bits. However, only order-1/2 OSD processes are usually utilized due to the rapid increase in the number of combinations. The order- $k$ OSD process can achieve the MLD performance, but the computation complexity of $2^{k}$ is too high, which makes the order- $k$ OSD impractical. The analysis of the relationship between the order of the OSD process and the error performance is given in [6]. However, it is difficult to apply the analysis to the turbo codes, which use the random interleavers between the two component RSC codes. Usually, the order-1/2 OSD is utilized, which offers a better tradeoff between the error performance and decoding complexity.

\section{Simulation Results}

Simulation studies are performed with the LTE turbo codes in additive white Gaussian noise (AWGN) channel with binary

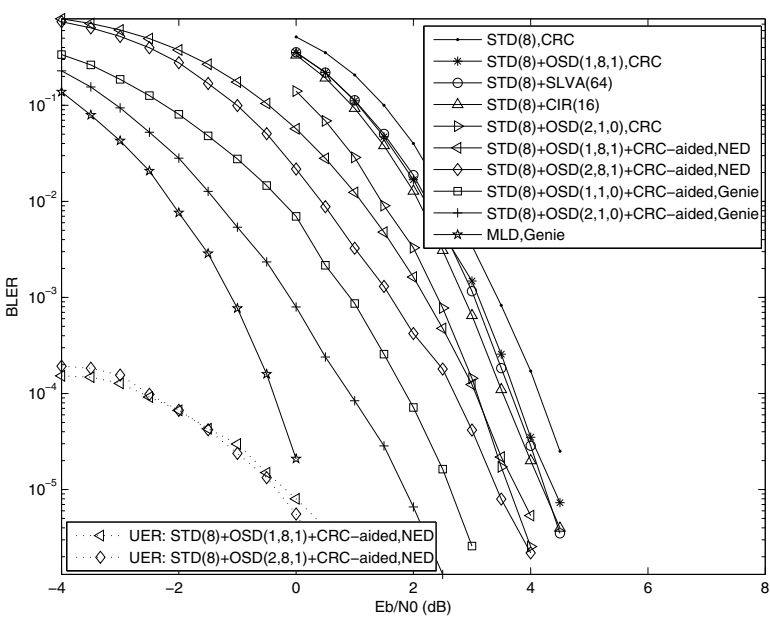

Fig. 1. Error performance of turbo-CRC code with $k=40$ by using different decoding algorithms and error detection criteria, $\eta=0.2$.

phase shift keying (BPSK) modulation. The enhanced MaxLog-MAP algorithm with the scaling factor of 0.75 over the extrinsic information [11] is applied as the $\operatorname{STD}(T)$, where the parameter $T$ is the maximum number of iterations. The $\operatorname{OSD}(N, f, \alpha)$ denotes the order- $N$ OSD process carried out in all the subsequent iterations from the $f$-th iteration, where the factor $\alpha$ is used for the LLR accumulation. For comparison, the performance of $\operatorname{SLVA}(L)$ and $\operatorname{CIR}(R)$ is also simulated, where the parameters $L$ and $R$ denote the number of the decoding paths in SLVA and the number of the repeated decoding in CIR, respectively. With the 24 CRC bits and the additional 12 tail bits of the turbo codes, the actual rates of the turbo-CRC codes are $(k-24) /(3 k+12)$, which are used to calculate the $E_{b} / N_{0}$ in the simulation results.

In the figures, the BLER is computed on the $m$ information bits only, by comparing them before encoding and after decoding. The undetected error rate corresponds to codewords which pass the error detection test (CRC or NED), but their $m$ information bits are wrong. In addition, any false alarm detection is also counted as an error and increase the BLER.

Fig. 1 shows the performance of the turbo-CRC codes with $k=40$ using different decoding algorithms. For the hybrid decoding with order-1 OSD applied to $\mathbf{G}^{\text {turbo }}$ after the last iteration using CRC detection (' $\operatorname{STD}(8)+\operatorname{OSD}(1,8,1)$, CRC'), only about $0.3 \mathrm{~dB}$ gain can be obtained over the STD(8); with the order-2 OSD process applied to $\mathbf{G}^{\text {turbo }}$ after each iteration ('STD $(8)+\mathrm{OSD}(2,1,0), \mathrm{CRC}$ '), about $1 \mathrm{~dB}$ gain is obtained over the $\operatorname{STD}(8)$. The SLVA(64) and the $\operatorname{CIR}(16)$ aided $\operatorname{STD}(8)$ only slightly outperform the ('STD $(8)+O S D(1,8,1), C R C$ '). The CRC-aided hybrid decoding with the order-2 OSD applied to $\mathbf{G}$ only after the last iteration (' $\operatorname{STD}(8)+\operatorname{OSD}(2,8,1)+\mathrm{CRC}$-aided, NED') can obtain $2 \mathrm{~dB}$ gain over the $\operatorname{STD}(8)$, which is much better than the hybrid decoding with order-2 OSD applied to $G^{\text {turbo }}$. In order to further show the decoding gain of the CRC-aided hybrid decoding, we present the performance of the CRCaided hybrid decoding applied to $\mathbf{G}$ with genie detection ('STD $(8)+\operatorname{OSD}(2,1,0)+C R C$-aided, Genie'), which can ap- 


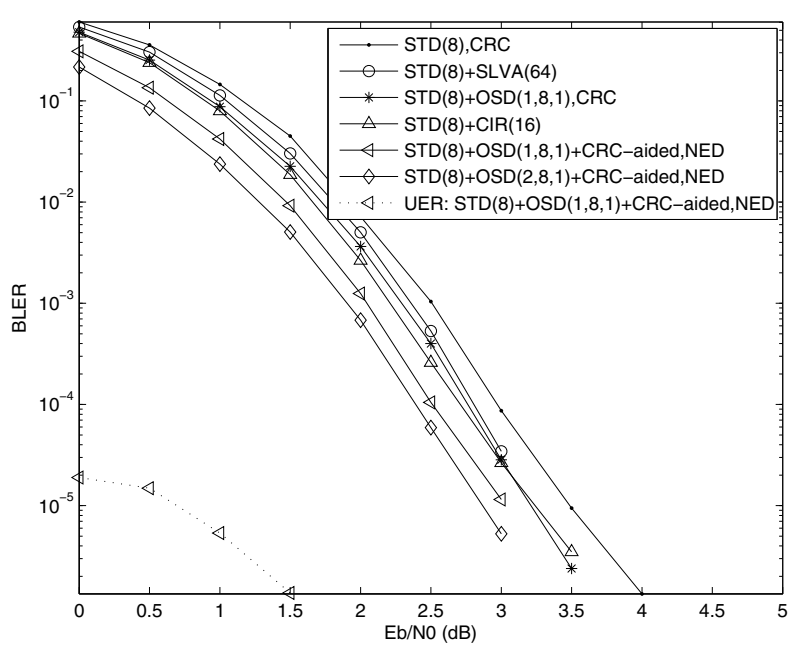

Fig. 2. Error performance of turbo-CRC code with $k=96$ by using different decoding algorithms and error detection criteria, $\eta=0.15$.

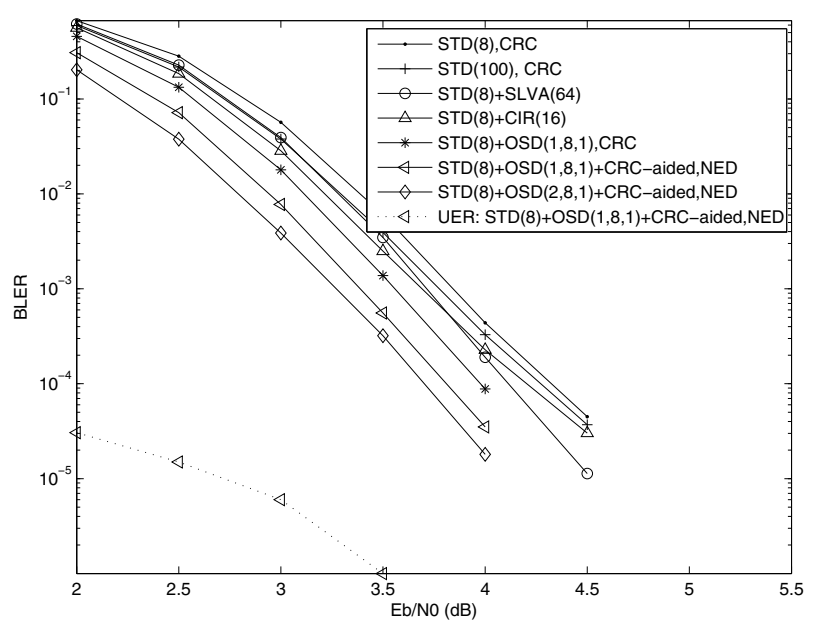

Fig. 3. Error performance of turbo-CRC code $k=352$ by using different decoding algorithms and error detection criteria, $\eta=0.025$.

proach the performance of the MLD within $1 \mathrm{~dB}$ at a BLER of $10^{-3}$, where the MLD is carried out by the exhaustive calculation of the Euclidean distances between the received vector and all the possible $2^{16}$ codewords. It can be seen that when we use the proposed NED detection with $\eta=0.2$, the UERs (dotted lines) are always lower than $10^{-3}$.

Fig. 2 and Fig. 3 show the error performance of the turboCRC code with $k=96$ and $k=352$ respectively. Although the overhead due to the CRC codes decrease with the increase of the $\mathrm{CB}$ length $k$, there is also a noticeable performance gain with the proposed scheme. For $k=96$, the CRC-aided hybrid decoding with the order-1 OSD applied to $\mathbf{G}$ only after the last iteration ('STD $(8)+\operatorname{OSD}(1,8,1)+\mathrm{CRC}$ aided, NED') has almost $0.5 \mathrm{~dB}$ and $0.2 \mathrm{~dB}$ performance gain over the $\operatorname{STD}(8)$ and the $\operatorname{CIR}(16)$ aided $\operatorname{STD}(8)$, respectively. When the CB length of the turbo code increases to $k=352$, our proposed decoding still has a considerable advantage over the STD for the punctured rate-3/4 turbo code, which is usually used for the hybrid automatic repeat request. The CRC-aided hybrid decoding ('STD (8)+OSD $(1,8,1)+\mathrm{CRC}$ aided, NED') applied to $\mathbf{G}$ obviously outperforms the hybrid decoding without CRC-aided and achieves almost $0.4 \mathrm{~dB}$ performance gain over the STD with 100 iterations ('STD(100)'). The UERs of the CRC-aided hybrid decoding with NED detection for both cases are lower than $10^{-5}$ when the BLER is $10^{-2}$, which are acceptable for many applications such as VoIP.

\section{CONCLUSION}

In this letter, a CRC-aided hybrid decoding algorithm is proposed, in which the CRC bits are not utilized for error detection but for error correction in the OSD process. An error detection criterion based on the normalized Euclidean distance is also proposed to compensate for the invalidation of CRC in error detection. Simulation results show that our proposed scheme can significantly improve the performance of the turbo-CRC codes with short information length, while keeping the undetected error rates sufficient low for many applications in wireless communication systems.

\section{REFERENCES}

[1] 3GPP, "TS36.212v10.2.0: multiplexing and channel coding," Jun. 2011.

[2] M. Muhleisen and B. Walke, "Evaluation and improvement of VoIP capacity for LTE," in Proc. 2012 European Wireless Conference, pp. 1-7.

[3] Y. Ould-Cheikh-Mouhamedou, S. Crozier, K. Gracie, P. Guinand, and P. Kabal, "A method for lowering turbo code error flare using correction impulses and repeated decoding," in Proc 2006 Int. Symp. Turbo. Codes and Related Topics.

[4] K. R. Narayanan and G. L. Stuber, "List decoding of turbo codes," IEEE Trans. Commun., vol. 46, no. 6, pp. 754-762, Jun. 1998.

[5] R. Wang, W. Zhao, and G. Giannakis, "CRC-assisted error correction in a convolutionally coded system," IEEE Trans. Commun., vol. 56, no. 11, pp. 1807-1815, Nov. 2008.

[6] M. P. C. Fossorier and S. Lin, "Soft-decision decoding of linear block codes based on ordered statistics," IEEE Trans. Inf. Theory, vol. 41, no. 5, pp. 1379-1396, Sep. 1995.

[7] M. Isaka, M. Fossorier, and H. Imai, "On the suboptimality of iterative decoding for turbo-like and LDPC codes with cycles in their graph representation, IEEE Trans. Commun., vol. 52, no. 5, pp. 845-854, May 2004.

[8] S. Gounai and T. Ohtsuki, "Decoding algorithms based on oscillation for low-density parity check codes," IEICE Trans. Fund., vol. E88-A, no. 8, pp. 2216-2226, Aug. 2005.

[9] M. Jiang, C. Zhao, E. Xu, and L. Zhang, "Reliability-based iterative decoding of LDPC codes using likelihood accumulation," IEEE Commun. Lett., vol. 11, no. 8, pp. 677-679, Aug. 2007.

[10] M. P. C. Fossorier, "Iterative reliability-based decoding of low-density parity check codes," IEEE J. Sel. Areas Commun., vol. 19, no. 5, pp. 908-917, May 2001.

[11] K. Gracie, S. Crozier, and A. Hunt, "Performance of a low-complexity turbo decoder with a simple early stopping criterion implemented on a SHARC processor," in Proc. 1999 Int. Mobile Sat. Conf., pp. 281-286. 